\title{
'Frontiers' grants count cost of success
}

[MUNICH] Ten years after a shaky start, the Human Frontiers Science Program (HFSP) has established itself as one of the leading intercontinental research grant programmes, and as an increasingly important source of funds for collaborative research in molecular biology and neuroscience.

But, as the programme celebrates its birthday, the HFSP board is struggling with its future financing. (The first celebration took place last December in Tokyo, and the final one will be in December in Washington - with a ceremony and two scientific workshops in Strasbourg, France, this week.)

At present, only one in eight applications for research grants and one in four applications for long-term fellowships can be approved for funding. Some of the best are refused. "We can only afford to support around half of the applications which our referees tell us are very high priority," says Piergiorgio Strata, professor of neurosciences at the University of Turin and a member of the HFSP board of trustees.

Akiyoshi Wada, director of the RIKEN Genomic Sciences Centre and one of the principal architects of HFSP, confirms that "the situation will have to change to be able to continue funding promising areas of biological research".

Human Frontiers was launched at the initiative of the Japanese government in 1989 , and has distributed US\$414 million to researchers around the world. Initially, however, it was widely distrusted for being motivated as much by political as scientific considerations.

The feeling was particularly strong among research groups in Europe and the United States who felt that Japan - which had a relatively poor track record in basic research had set up the programme to exploit their

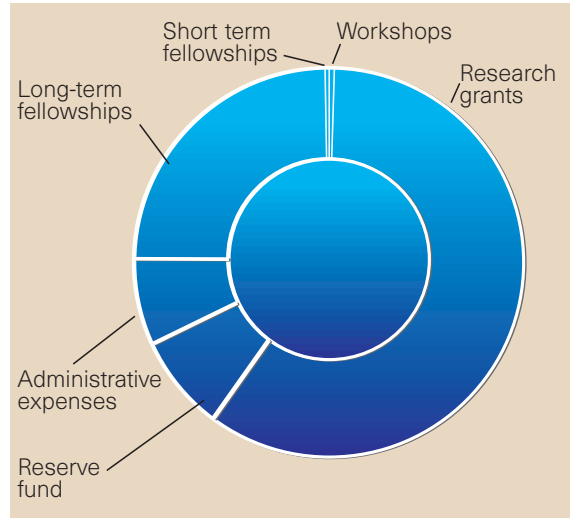

The HFSP has spent a cumulative budget of US\$414 million since its launch in 1989.

Research grants average US $\$ 230,000$, and teams receiving them must have an international make-up. Eighty per cent of the 422 awarded so far involve scientists from at least two continents, and a third involve three continents.

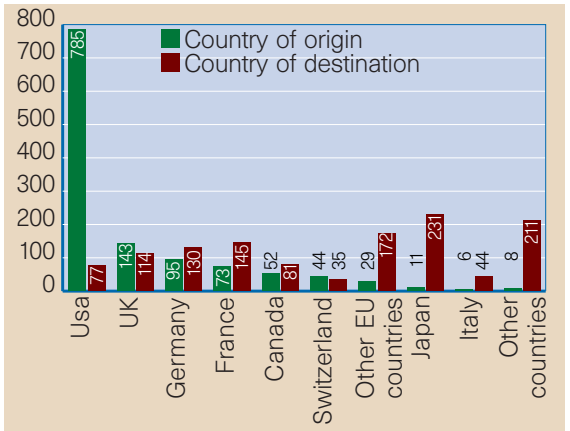

HFSP long-term fellows: where they come from and where they go.

work. But this attitude has been "completely reversed" after ten years of genuine support for international research, says Wada.

"There were several misunderstandings in the past, which led people to think that HFSP was an attempt by Japan to promote its technological development," says Wada. "Fortunately the concept of the programme - to support collaborative basic research through a common funding mechanism has now been fully understood."

By the end of the month, the Human Frontiers board is expected to select a successor to Michel Cuénod, who steps down as secretary general next spring. There are three candidates, all internationally renowned scientists. One is Torsten Wiesel (75), Nobel laureate in 1981 for work on the neurophysiology of vision, and currently president emeritus of the Rockefeller University in New York.

The second is Albert Aguayo (65), a professor of neuroscience at McGill University in Montreal, Canada. Aguayo, a native of Argentina, works on neuron regeneration and is a former chairman of the Human Frontiers council of scientists. The third candidate is Lennart Philipson (70), a molecular biologist at the Karolinska Institute in Stockholm who formerly headed the European Molecular Biology Laboratory (EMBL) in Heidelberg, Germany.

The programme has become one of biology's best-regarded grants schemes. But its success has led to oversubscription, and the board remains keen to increase the budget to raise the success rate for applicants.

One of the main tasks facing the next secretary general will at the same time be to redress the continuing funding imbalance between Japan and its partners. Japan provided nearly all the funds for the first three years of the programme and has contributed 80 per cent since.

But the scientific benefits reaped by Japan remain lower than those of other partners. Within the research grants section, for example, which absorbs a third of the budget, 32 per cent of investigators have come from the
United States, and only 16 per cent from Japan. Yet the United States will contribute less than 10 per cent to the budget next year.

A new basis for funding was proposed in 1997. Partner states — apart from Japan agreed in principle to increase their contributions over five years, up to a level related to their gross national product (see Nature 387, $446 ;$ 1997). Japan's contribution would remain constant until it fell to 50 per cent of the total.

The new formula would increase the annual budget from $\$ 45$ million in 1997 to $\$ 60$ million in 2002. But few countries are on target to meet the non-binding increases. Already the 1999 budget of $\$ 47$ million falls well short of the promised $\$ 53$ million.

The United States wins out in the longterm fellowship programme, as well as in the research grants programme (see left). While the United States remains a Mecca for young scientists from other countries, its own researchers are less convinced of the benefits of doing a postdoc abroad.

The stature of the United States gives its scientists little incentive to leave, says James Sutherland, a mouse geneticist working as a Human Frontiers fellow at the EMBL outstation at the Adriano Buzzati-Traverso campus at Monterotondo near Rome.

Sean Oldham, a developmental geneticist from North Carolina, now working at the University of Zurich, says he applied for a Human Frontiers fellowship because of its prestige value - but most colleagues told him it would be a "retrograde career step to go to Europe".

Oldham says his colleagues felt he would find it harder to get a job in the United States, because of loss of contact with US research networks, and because a European postdoc would not be taken seriously. Scientists, he was told, "do not work hard in Europe".

But Oldham says that the stereotype of Europe as a scientific playground is false. Both he and Sutherland believe that, provided they maintain contacts with US scientists, they will have no trouble returning to the US academic system.

"Working in a foreign laboratory broadens your horizons - your personal human frontiers - enormously," says Oldham. It is valuable to learn that there are other successful research cultures apart from the United States, he says.

For example, he has been impressed by "the extraordinary meticulousness" of Swiss researchers, but he has been taken aback by "the extraordinary respect that Swiss scientists maintain for the authority of the professor". Overall it is different, and it works, he says: "After all, Switzerland has the highest density of Nobel prizewinners in the world." Alison Abbott\& Asako Saegusa 